\section{Getting the dose right for obese children}

\author{
Brian J Anderson, ${ }^{1}$ Nick HG Holford ${ }^{2}$
}

A survey conducted by Collier et $a l^{1}$ highlights the problem of drug dosing in obese children. The authors identify two aspects that require greater attention from paediatric practitioners: the identification of the obese child and the lack of use of ideal body weight (IBW) for drug calculation in that obese child. Both involve effort from prescribers to go beyond simply measuring total body weight (TBW). There remains a question about the benefits of using IBW compared with using TBW or any other measure of body mass. The lack of enthusiasm to calculate IBW obvious from the Collier et al survey is reflective of uncertainty concerning dose calculation in the obese child.

Collier et al propose anecdotally that using IBW rather than TBW would have avoided toxicity from an overdose of aminophylline. While we agree that aminophylline maintenance doses using TBW may be over-predicted in obesity, initial dosing based on either IBW or TBW for a target concentration (TC) of $10 \mathrm{mg} / \mathrm{L}$ is unlikely to be toxic. ${ }^{2}$ While the use of TBW to calculate initial doses may not be ideal for the maintenance dose, the problem could have been avoided by measuring theophylline concentrations to individualise the dose.

We will not deal here with the challenge of choosing a TC but rather with what is an appropriate size metric to predict doses to achieve a desired TC. Rational dose calculation uses the volume of distribution (V) and clearance (CL) with the TC required to achieve the desired effect. ${ }^{3}$

$$
\text { Loading Dose }=\mathrm{V} \times \mathrm{TC}
$$

Maintenance Dose Rate $=\mathrm{CL} \times \mathrm{TC}$

${ }^{1}$ Department of Anaesthesiology, University of Auckland, Auckland, New Zealand; ' 2 Department of Pharmacology \& Clinical Pharmacology, University of Auckland, Auckland, New Zealand

Correspondence to Professor Brian J Anderson, Department of Anaesthesiology, University of Auckland, Auckland 1023, New Zealand; briana@adhb.govt.nz
Other size descriptors besides IBW and TBW have been put forward for use in the obese patient, for example body surface area and lean body mass (commonly used interchangeably with lean body weight and fat free mass (FFM)). As Gal $e t a l^{2}$ pointed out for aminophylline, the descriptor required to predict $\mathrm{V}$ may not be the same as that required to predict CL, which adds further complexity to dose calculations. Few studies have attempted to distinguish between the effects of obesity and those due to differences in size for both $\mathrm{V}$ and CL.

Body mass index (BMI) may be used as an indication of obesity but it is uncommonly used to predict dose in children or adults because it fails to distinguish between adipose tissue and lean muscle mass. Further, Collier et al ${ }^{1}$ correctly point out that BMI in children must be interpreted with reference to age and sex. Consequently, IBW has been proposed as the preferred metric for maintenance dosing of a number of drugs. However, it is not the best metric for all drugs. It may not even be the best metric for those listed by Collier et al. ${ }^{1}$

Size metrics have been investigated in anaesthesia where drugs often have a low therapeutic index. Getting the dose right is important. The search for the 'best' body size predictor has revealed that more mass than expected from IBW may be required for some drugs. The addition of $40 \%$ of the excess weight to the IBW for propofol infusion calculation has been suggested. Rocuronium dosing improved when 20\% of the excess weight was added to IBW. Both V and CL of the popular anaesthetic drug propofol in obese children and adults and non-obese adults and children were best predicted using TBW as the size descriptor. ${ }^{4}$ Thus IBW is not necessarily the best size metric for all drugs in obese children.

The idea of adding a fraction of fat mass to FFM has been used to estimate the mass that best describes structure and function based on allometric scaling theory. ${ }^{3}$ This mass has been called normal fat mass (NFM). NFM is calculated from FFM and FAT mass (ie, FAT equals TBW minus FFM). The fraction of FAT that contributes to the structural (V) or functional (CL) size, Ffat, is specific to each drug

$$
\mathrm{NFM}=\mathrm{FFM}+\text { Ffat } \times \text { FAT }
$$

If Ffat is estimated to be zero then FFM alone predicts size while if Ffat is 1 then size is predicted by TBW. The use of NFM based on allometric theory and partition of body mass into fat and fat-free components provides a principle-based approach applicable to predicting size and body composition effects on pharmacokinetics of all drugs in babies, children and adults.

Collier $e t a l^{1}$ justifiably raise the important issue of dosing in the obese child. However, getting the dose right may be more than simply learning how to calculate IBW. We recommend the use of FFM rather than IBW to avoid confusion between methods used in children and adults. The reason for differences in size metrics which may occur for CL and V is not yet known so we must currently rely upon pharmacokinetic studies that have explicitly determined the appropriate size metric(s) for these key parameters or failing that continue to use an empirical approach. While drug lipophilicity may be a factor determining drug disposition, this remains untested. The contribution from body fat is a factor to consider in the disposition of all drugs. While the empirical use of IBW may prove better than TBW for some drugs, such as those listed by Collier et al,${ }^{1}$ theory based metrics have not been investigated for these drugs. The use of a theory-based metric such as NFM is a more flexible way of accounting for weight and obesity. NFM requires the determination of Ffat which has so far only been determined for a handful of drugs. ${ }^{5}$ Once we have greater understanding of the principles determining Ffat then it may be possible to predict this parameter using physiological based pharmacokinetic models. This area of research requires further clarification before paediatricians can demonstrate that they can dose every drug appropriately in the obese child. 
Competing interests None declared.

Provenance and peer review Commissioned; internally peer reviewed.

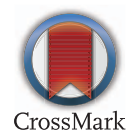

To cite Anderson BJ, Holford NHG. Arch Dis Child 2017;102:54-55.

Received 27 July 2016

Revised 25 August 2016

Accepted 27 August 2016

Published Online First 21 September 2016
Arch Dis Child 2017;102:54-55

doi:10.1136/archdischild-2016-311696

\section{REFERENCES}

1 Collier H, Nasim M, Ganhi A. Prescribing in obese children-how good are paediatricians. Arch Dis Child 2017;102:61-2.

2 Gal P, Jusko WJ, Yurchak AM, et al. Theophylline disposition in obesity. Clin Pharmacol Ther 1978:23:438-44.

3 Anderson BJ, Holford $\mathrm{NH}$. Understanding dosing: children are small adults, neonates are immature children. Arch Dis Child 2013:98:737-44.
4 Cortínez LI, Anderson BJ, Penna A, et al. Influence of obesity on propofol pharmacokinetics: derivation of a pharmacokinetic model. Br J Anaesth 2010;105: 448-56.

5 Holford NHG. Using normal fat mass to account for body size and composition. WCOP, 2016.

http://abstracts-wcoprhcloudcom/wp-content/uploads/ 2016/08/Using-normal-fat-mass-to-account-forbody-size-and-composition.pdf (accessed 25 Aug 2016). 